This is the peer reviewed version of the following article: Tuffrey-Wijne, Irene, Curfs, Leopold, Finlay, Ilora and Hollins, Sheila (2019) "Because of his intellectual disability, he couldn't cope." Is euthanasia the answer? Journal of Policy and Practice in Intellectual Disabilities, 16(2), pp. 113-116., which has been published in final form at https://doi.org/10.1111/jppi.12307. This article may be used for non-commercial purposes in accordance with Wiley Terms and Conditions for Use of Self-Archived Versions. 


\title{
"Because of his intellectual disability, he couldn't cope." Is euthanasia the answer?
}

\author{
Authors \\ Irene Tuffrey-Wijne Kingston \& St George's University, UK \\ Leopold Curfs Governor Kremers Centre, Netherlands \\ Ilora Finlay Cardiff University, UK \\ Sheila Hollins St George's University, UK
}

\section{Key words}

Euthanasia

Intellectual disabilities

Autism spectrum disorders

Legislation

Netherlands

\begin{abstract}
In 2018, the authors published their analysis of 9 online case reports by the Euthanasia Review Committee in the Netherlands, involving people with intellectual disability and/or autism spectrum disorder who were given euthanasia. In this commentary and update on that work, they reflect further on the challenges of assessing "unbearable suffering without prospect of improvement", which is one of the Dutch legal due care criteria. Two more recent case reports are presented in detail, where doctors struggled to assess and sometimes came to divergent conclusions. In both cases, limitations resulting from the intellectual disability and autism spectrum disorder were seen by physicians as causes of unbearable suffering, leading them to agreeing to the patient's euthanasia request. The authors discuss their concern about the implications of accepting the effects of lifelong disability as reasons for euthanasia, not only for individuals but for society as a whole.
\end{abstract}


Tuffrey-Wijne I, Curfs L, Finlay I, Hollins S

"Because of his intellectual disability, he couldn't cope." Is euthanasia the answer?

26/04/2019 Accepted for publication in Journal of Policy and Practice in Intellectual Disabilities

\section{Euthanasia in the Netherlands}

In 2017, 4.4\% of deaths in the Netherlands (more than 1 in 25) were physician-assisted deaths. Most of these (96\%) were deaths by euthanasia, where a physician administers a lethal injection, rather than physician-assisted suicide, where patients take the lethal medicine themselves. This means that almost everyone (including many people with intellectual disabilities) will know about euthanasia through immediate experience within their own circle of family and friends. In the Netherlands, euthanasia is socially accepted. It is talked about as a viable, indeed sometimes preferable, way to die.

We investigated the effects of Dutch euthanasia legislation on people with intellectual disabilities and autism spectrum disorders. We are in the unique position of not only having significant clinical and academic expertise in the fields of intellectual disability and palliative care, but also sharing between us a good understanding of the languages and the cultures of both the Netherlands and the UK - two countries who have translated the key ethical principles of autonomy, beneficence, nonmaleficence and justice into divergent legal frameworks. We were helped by the laudable transparency of the Dutch system, where all cases of euthanasia must be reported to a Euthanasia Regional Review Committee (RRC), as described in Reinders et al.'s position paper on Eugenics. The task of the RRC is to scrutinise whether six legal "due care criteria" are met (Regional Euthanasia Review Committees, 2019a). The RRC not only produces an annual report, it also publishes a selection of case reports online.

We searched the RRC online database for cases of euthanasia involving people with intellectual disability and/or autism spectrum disorder. Between 2012 and 2016, there were 25,930 notifications of euthanasia or assisted suicide; 416 case reports were put online (in Dutch only, although a handful have now been translated into English and put onto the English version of the RRC website). We found six case reports of people with intellectual disability and three of people with autism spectrum disorder. We published our analysis of these reports last year (Tuffrey-wijne, Curfs, Finlay, \& Hollins, 2018); our study is briefly described in the longer version of the Eugenics position paper.

A range of challenging issues arose from our analysis, including the difficulties in assessing whether the patient had made a "voluntary and well-considered request" (one of the legal due care criteria), which is closely linked to an assessment of the patient's decision-making capacity. We had serious concerns about the apparent lack of stringency in these assessments.

In this commentary on the Eugenics position paper, however, we would like to focus on the second of the legal due care criteria, "unbearable suffering without prospect of improvement", which is the cornerstone of Dutch euthanasia legislation. This requirement poses a key challenge in relation to euthanasia requests from people with intellectual disabilities and/or autism spectrum disorder, particularly if the nature of suffering for which euthanasia is sought is related to, or affected by, their disability.

For this commentary, we have updated our online search of the Dutch database. In 2017 and 2018, 161 online case reports included four cases of people with intellectual disabilities, one of whom also had autism spectrum disorder; and three further cases of people with autism spectrum disorder (Regional Euthanasia Review Committees, 2019b). We will present two of these cases as the basis of 
Tuffrey-Wijne I, Curfs L, Finlay I, Hollins S

"Because of his intellectual disability, he couldn't cope." Is euthanasia the answer?

26/04/2019 Accepted for publication in Journal of Policy and Practice in Intellectual Disabilities

our discussion. A full English translation of the case reports is available from the corresponding author on request.

\section{Unbearable suffering without prospect of improvement}

The RRC gives the following guidance:

"The patient's suffering is considered to be without prospect of improvement if the disease or disorder causing the suffering is incurable and there are no means of alleviating the symptoms so that the suffering is no longer unbearable." (Regional Euthanasia Review Committees, 2019a)

It is important to note the absence of any description of the causes of suffering for which euthanasia is allowed, although a 2002 court case and subsequent RRC verdicts have made it clear that the suffering must be the result of a medical condition. This can be either somatic or psychiatric; it can also be an accumulation of conditions related to old age. There is no mention of life expectancy, opening the door to euthanasia for people who could have lived for many more decades. Two of the people with autism spectrum disorder (but without intellectual disabilities) were aged between 18 and 30 (case 2017-80 and 2018-24). They died from euthanasia after their physicians agreed that their suffering, stemming from the autism spectrum disorder itself, was unbearable and could not be relieved.

There has been much debate around the ever-widening parameters of what constitutes "suffering" with regards to the Dutch legal due care criteria, as well as the lack of the need for a short life expectancy. The debate, and indeed fierce criticism, has centred particularly on the growing number of cases of euthanasia for people with non-life-threatening psychiatric conditions. However, it can be argued that this widening of scope is inevitable, or at least logical. If the purpose of assisted dying legislation is to relieve suffering, then it could be seen as illogical and inequitable to allow euthanasia for some kinds of suffering but not others. Psychological suffering can be as unbearable as physiological pain. It is also worth noting that even people with terminal somatic conditions do not give pain as the main reason for wanting euthanasia, but state overwhelmingly that they suffer most from functional limitations, dependence on others and a reduced ability to engage in enjoyable activities (Oregon Public Health Division, 2018).

What is not made specific within the legislation and guidelines is whether the "unbearable suffering" that must underpin a euthanasia request may be the result of a lifelong disability, including the effects of an intellectual disability or autism spectrum disorder. The following case summaries highlights the difficulties doctors have in assessing whether the lifelong nature of such limitations is indeed a valid reason for approving a euthanasia request. In reading these report, it is important to note that the Dutch due care criteria include the need for the doctor to consult at least one other, independent physician, although there is no obligation to have a consensus, and the advice of the independent consultant can be disregarded. 
Tuffrey-Wijne I, Curfs L, Finlay I, Hollins S

"Because of his intellectual disability, he couldn't cope." Is euthanasia the answer?

26/04/2019 Accepted for publication in Journal of Policy and Practice in Intellectual Disabilities

\section{Case 2018-69}

The patient was a man in his 50s with intellectual disabilities. He had been given a diagnosis 30 years previously of "borderline state in an autistic, socially isolated, obsessive personality of a prepsychotic man". He had functioned quite poorly within society, but had managed to cope without professional help, thanks to the structure and support in his life.

Three years before the euthanasia, he was referred to mental health services following the death of one of his parents. The professionals diagnosed a grief response in a vulnerable man with autistic and psychotic characteristics, for whom a heavy life burden could trigger depression. Over the following years, he had two brief in-patient episodes because of suicidal tendencies. He received various forms of treatment and support, including medication, sessions with a psychiatrist, and support from a community mental health nurse. He started doing voluntary work, creative therapy and psycho-education. All this helped, but didn't diminish his perceived burden of suffering. His psychiatrist concluded that further psychiatric treatment was unlikely to help, but he might benefit from supported living in a setting that offered structure and care. There was a waiting list, but the patient eventually moved to such a setting, nine months before his death. However, the forced interactions with other people meant that this didn't relieve his suffering either.

The patient had previously asked his GP for euthanasia, but his GP did not agree to his request. Two years before his death, the patient went to the End of Life Clinic (which offers support with euthanasia trajectories for people whose GP has turned them down). His request was initially rejected by a nurse from the clinic, due to the short time frame since his parent's death. A year later, he referred himself to the clinic again. Nine months before his death, he met with one of the clinic's physicians (who eventually carried out the euthanasia). This doctor met him four more times in the three months before the death. The doctor also consulted his GP, his psychiatrist, his mental health nurse and his support worker.

The doctor then consulted a psychiatrist for a second opinion with regards to the diagnosis and treatment options. This psychiatrist concluded that autism spectrum disorder was the primary diagnosis, for which there were hardly any treatment options. He suggested treatment for his depressive or psychotic symptoms, but the patient refused this. The doctor was similarly of the opinion that the patient's suffering was caused almost exclusively by his autism spectrum disorder. Treatment of any other conditions would make little difference to his suffering.

This suffering was described as follows: everything was too much for the patient; he had nightmares and panic attacks; he was overstimulated, particularly through his interactions with other people. After the death of one of his parents, he was suffering due to an increased dependence on carers. His inflexible and compulsive way of coping meant that he couldn't adapt to constantly changing people in his environment. He had lost the overview of his daily life. He felt powerless in his inability to function in today's society, and was not the person he wanted to be, with a job and a family. He experienced his suffering as unbearable. His doctor agreed.

As part of the euthanasia trajectory, the doctor consulted another independent psychiatrist for a second opinion on whether the patient met the due care criteria. This consultant saw the patient twice. He found the patient strongly pre-occupied with his death wish, but unable to explain the nature of his suffering, or why it was so hopeless. The consultant found that the patient did have 
Tuffrey-Wijne I, Curfs L, Finlay I, Hollins S

"Because of his intellectual disability, he couldn't cope." Is euthanasia the answer?

26/04/2019 Accepted for publication in Journal of Policy and Practice in Intellectual Disabilities

decision-making capacity, but was unable to judge the available alternatives and possibilities. The consultant thought that improvements might be possible, and stated that an inability or reluctance to accept help does not justify euthanasia. He concluded that the due care criteria were not met.

The doctor disagreed with this conclusion. He was of the opinion that the patient's inability to consider alternatives or accept help was in fact part of his condition that caused him such suffering, and this was impossible to relieve. The psychiatrist's report did not change the doctor's mind, and she carried out the euthanasia.

The RRC questioned this. The doctor explained that she herself was in no doubt about the unbearable nature of the patient's suffering, nor of the lack of prospect of improvement; that she had not wanted to burden the patient with further assessments; and that she did not want to give the impression of "doctor shopping". The RRC found that the consultant's report was too brief and inadequately argued, and the doctor should have sought another second opinion. The RRC concluded that the doctor had not acted in accordance with the due care criteria, and referred the case to the Board of Procurators General and the General Health Care Inspectorate.

\section{Case 2018-27}

A man in his 60s with a mild intellectual disability had, for the past five years, been suffering from unexplained severe pains that started in his abdomen and radiated to his back and legs. He had undergone very many investigations and treatments, including medication, surgery and therapies, to no avail. Cognitive behavioural therapy and psychotherapy were not considered to be worthwhile, due to the patient's limited intelligence. He had abandoned several treatments, turned down suggestions and refused further investigations. He asked his GP for euthanasia.

The first independent physician who assessed the euthanasia request found that the nature of the patient's suffering was "subjective" to a higher-than-average degree - in other words, it was less "palpable" to the physician than usual. He stated, however, that the burden of suffering should be seen in light of the patient's limited coping ability, resulting from his intellectual disability and almost complete inability to reflect on his situation. This physician concluded that the man's suffering did not have a demonstrable somatic cause, nor was it based on psychiatric illness. He concluded, therefore, that the due care criteria were not met, and euthanasia would contravene the 2002 court case verdict that specified the need for an underlying classifiable medical condition.

The GP then consulted a second independent physician, a psychiatrist, who diagnosed a somatoform disorder, possibly influenced by traumatic experiences in early life. The psychiatrist concluded that the patient had become so preoccupied with his physical condition that it had become part of his identity. This, combined with comorbid depressive symptoms, made for a poor outlook. The psychiatrist thought there were no realistic treatment options for the patient.

At the GP's request, a third independent physician visited the patient 2,5 months before the death. This physician found a discrepancy between the descriptions the patient gave of his suffering, and his observable behaviour. The third physician saw no signs of tiredness or other suffering and was unable to say that there was "palpable" unbearable suffering. He also concluded that due to the 
Tuffrey-Wijne I, Curfs L, Finlay I, Hollins S

"Because of his intellectual disability, he couldn't cope." Is euthanasia the answer?

26/04/2019 Accepted for publication in Journal of Policy and Practice in Intellectual Disabilities

patient's limitations, his request was not "well-considered". The due care criteria, therefore, had not been met.

The GP consulted a fourth independent physician, who was a geriatric psychiatrist, who visited the patient a week before the death. She diagnosed a classifiable psychiatric condition causing suffering without prospect of improvement, with no reasonable treatment options. She dismissed the first physician's concern about contravening the 2002 court verdict.

The patient saw no future for himself. He only wanted to die, and he was utterly fixated on this wish. The GP finally concluded that the patient's pain was indeed unbearable, and that he possessed insufficient coping strategies to manage his symptoms. The patient's pain experience was largely psychological. This could be significantly affected by his dependent personality, his intellectual disability and his loneliness.

Following the euthanasia, the RRC's verdict was that the patient "could not cope with his complaints, due to his intellectual and cognitive limitations (...) This justifies the conclusion that the suffering was without prospect of improvement and that there were no alternatives." The RRC found that the GP had come to a reasonable conclusion, had handled with due care, and had acted within the law.

\section{How can "unbearable suffering" and "no prospect of improvement" be assessed?}

It is unusual for the RRC to pass a verdict of "due care criteria not met"; in 2017, this happened in only 12 out of 6,585 cases $(0.18 \%)$. Mostly, failure to meet the criteria is related to procedural failings, as in case 2018-69, where the doctor failed to secure an adequate second opinion. Doctors who did not meet the due care criteria are almost always cleared at the next stage; in fact, so far only one case has ever been referred to the prosecutors (in 2018, related to euthanasia of a patient with dementia).

The unbearable nature of the patient's suffering is not reviewed or questioned by RRC. The responsibility for assessing whether the patient's suffering is bad enough and hopeless enough to warrant euthanasia, rests solely with their physician. The obvious problem is that suffering is an unavoidably subjective concept. If the patient consistently states that he can no longer bear his suffering, how can any doctor refute this? There is a strong emphasis within Dutch society on the right to individual autonomy, and there are calls for a more accessible euthanasia route. But at present, the doctor being asked to give the lethal injection must assess and agree that the suffering is unbearable. The RRC Code of Practice stipulates that doctors must be able to empathise with the patient's suffering to such an extent that they can "feel" the suffering: it must be "palpable". The two cases show how difficult this is if the patient's suffering stems from disabilities that affect communication and social interaction. Some of the doctors involved in both cases raised concerns. In the other cases we studied, it was mostly accepted by doctors that suffering could consist of psychological pain, dependency, social isolation, loneliness and a lack of coping mechanisms that were a result of intellectual disability. There were also several examples of rigid thinking, where the patient was fixated on the idea of euthanasia and unable or unwilling to consider alternatives. In a society where, as we have seen, most citizens are aware that they can ask for euthanasia if they feel their suffering is hopeless, it is inevitable that people with intellectual disabilities can ask for it too; 
and as equal citizens, they have a right to do so. But the fact that the disability itself, rather than an acquired medical condition, can be accepted as a cause of suffering that justifies euthanasia is deeply worrying. Furthermore, disabled people reject the medical model of disability, arguing that disability is a social construction.

If the "unbearable suffering" does indeed result from living with the limitations of intellectual disability or autism spectrum disorder, then it is inevitable that there is "no prospect of improvement". This, too, is evident from the case reports. In cases of persistent treatment refusal, or persisting problems despite having tried many different approaches and treatments, physicians tend to reach the conclusion that euthanasia is the only remaining option for the patient. Reading the case reports, we are in no doubt that these patients did indeed suffer deeply and consistently. However, we know that people with disabilities experience severe inequalities in opportunities and in health and social care provision, which may well play a part in their lack of "prospect". Current society is not a level playing field, where everyone has a full range of life choices and can make autonomous choices about them. The bereaved man in case 2018-69 was able to live adequately for many decades, but society was unable to support him after his parent died. The difficulty with legalised euthanasia is that it becomes normalised, as we have seen. This makes it perhaps all too easy for people to request euthanasia, and to be granted such a death as a "way out" of painfully difficult situations and circumstances, rather than addressing underlying issues of inequality and a lack of adequate support for people with very complex needs. We are not convinced that euthanasia is a suitable solution in the cases we reviewed.

\section{References}

Oregon Public Health Division. (2018). Oregon Death with Dignity Act: Data summary 2017. Retrieved January 18, 2018, from http://www.oregon.gov/OHA/PH/ProviderPartnerResources/EvaluationResearch/DeathwithDi gnityAct/pages/index.aspx

Regional Euthanasia Review Committees. (2019a). Due care criteria. Retrieved January 18, 2019, from https://english.euthanasiecommissie.nl/due-care-criteria

Regional Euthanasia Review Committees. (2019b). Uitspraken en uitleg [Verdicts and explanations]. Retrieved January 18, 2019, from https://www.euthanasiecommissie.nl/uitspraken-en-uitleg

Tuffrey-wijne, I., Curfs, L., Finlay, I., \& Hollins, S. (2018). Euthanasia and assisted suicide for people with an intellectual disability and/or autism spectrum disorder: an examination of nine relevant euthanasia cases in the Netherlands (2012 - 2016). BMC Medical Ethics, 19(17), 1-21. 